\title{
The parallax, mass and age of the PSR J2145-0750 binary system
}

\author{
O. Löhmer ${ }^{1}$, M. Kramer ${ }^{2}$, T. Driebe ${ }^{1}$, A. Jessner ${ }^{1}$, D. Mitra ${ }^{1}$, and A. G. Lyne ${ }^{2}$ \\ 1 Max-Planck-Institut für Radioastronomie, Auf dem Hügel 69, 53121 Bonn, Germany \\ e-mail: loehmer@mpifr-bonn.mpg.de \\ ${ }^{2}$ University of Manchester, Jodrell Bank Observatory, Macclesfield, Cheshire SK11 9DL, UK
}

Received 6 April 2004 / Accepted 22 June 2004

\begin{abstract}
We present results of high-precision timing measurements of the binary millisecond pulsar PSR J2145-0750. Combining 10 yrs of radio timing data obtained with the Effelsberg 100-m radio telescope and the Lovell 76-m radio telescope we measure a significant timing parallax of 2.0(6) mas placing the system at $500 \mathrm{pc}$ distance to the solar system. The detected secular change of the projected semi-major axis of the orbit $\dot{x}=1.8(6) \times 10^{-14} 1 \mathrm{t}-\mathrm{s} \mathrm{s} \mathrm{s}^{-1}$, where $x=\left(a_{\mathrm{p}} \sin i\right) / c$, is caused by the proper motion of the system. With this measurement we can constrain the orbital inclination angle to $i<61^{\circ}$, with a median likelihood value of $46^{\circ}$ which is consistent with results from polarimetric studies of the pulsar magnetosphere. This constraint together with the non-detection of Shapiro delay rules out certain combinations of the companion mass, $m_{2}$, and the inclination, $i$. For typical neutron star masses and using optical observations of the carbon/oxygen-core white dwarf we derive a mass range for the companion of $0.7 M_{\odot} \leq m_{2} \leq 1.0 M_{\odot}$. We apply evolutionary white dwarf cooling models to revisit the cooling age of the companion. Our analysis reveals that the companion has an effective temperature of $T_{\text {eff }}=5750 \pm 600 \mathrm{~K}$ and a cooling age of $\tau_{\text {cool }}=3.6(2) \mathrm{Gyr}$, which is roughly a factor of three lower than the pulsar's characteristic age of 10.4 Gyr. The cooling age implies an initial spin period of $P_{0}=13.0(5) \mathrm{ms}$, which is very close to the current period.
\end{abstract}

Key words. astrometry - stars: neutron - stars: white dwarfs - stars: binaries: general - stars: pulsars: individual: PSR J2145-0750

\section{Introduction}

Millisecond pulsars (MSPs) have small spin periods $(\sim 1.5-30 \mathrm{~ms})$, small spin-down rates $\left(\dot{P} \lesssim 10^{-19} \mathrm{~s} \mathrm{~s}^{-1}\right)$, and are believed to be recycled by the accretion of mass from an evolving companion star (e.g. Alpar et al. 1982). About $~ 80 \%$ of the MSP population in the Galactic plane are members of binary systems. Most of those move in nearly circular orbits around low-mass companions, forming the class of low-mass binary pulsars (LMBPs). LMBPs have spin periods of $P \lesssim 10 \mathrm{~ms}$, small eccentricities $e \lesssim 10^{-3}$, and companions of mass $0.15 M_{\odot} \lesssim m_{2} \lesssim 0.4 M_{\odot}$, presumably helium (He)-core white dwarfs (WDs). The evolutionary history of LMBPs is well understood. During the giant phase of the $\sim 1 M_{\odot}$ companion star, mass overflows its Roche lobe and is accreted onto the neutron star (NS). The stable mass accretion causes the pulsar to spin up to millisecond periods and prevents a central helium ignition of the giant, resulting in a binary system with a recycled millisecond pulsar and a low-mass He-core WD (see review by Bhattacharya \& van den Heuvel 1991; Phinney \& Kulkarni 1994).

In the last decade, pulsar surveys have revealed a new class of binary pulsars, the intermediate-mass binary pulsars (IMBPs), with companion masses above $0.45 M_{\odot}$. Like in the LMBP case, the IMBPs have almost circular orbits and WD companions, but show larger spin periods $P \gtrsim 10 \mathrm{~ms}$ and slightly higher orbital eccentricities. As helium ignition in the giant core starts above a core mass of $0.45 M_{\odot}$ (Kippenhahn \& Weigert 1990), the companions have to be WDs with carbon/oxygen $(\mathrm{CO})$ or oxygen/neon/magnesium $(\mathrm{ONeMg})$ cores. PSR J2145-0750 is most probably a member of the class of IMBPs. Discovered by Bailes et al. (1994), the 16-ms pulsar is in a nearly circular orbit, with an orbital period of 6.8 days. The system shows some of the peculiar properties of the IMBP class: (1) The pulsar companion has a mass of $m_{2}>0.43 M_{\odot}$ and probably even larger than $0.51 M_{\odot}(50 \%$ C.L.), suggesting it to be a CO-core WD. From optical observations of the PSR J2145-0750 system and using the dispersion measure (DM) distance of $500 \mathrm{pc}$, Lundgren et al. (1996) concluded that the companion is a WD. This is confirmed by our measurement of a timing parallax (see Sect. 4.1) showing that the DM distance is the true distance of the pulsar. (2) PSR J2145-0750 has a spin period of $16 \mathrm{~ms}$, which is considerably longer than the average period of $\sim 4 \mathrm{~ms}$ of LMBPs. (3) The surface magnetic field of the pulsar is quite high: $B_{\mathrm{S}}=6 \times 10^{8} \mathrm{G}$. (4) Finally, the spin-down age of the pulsar of $\sim 10 \mathrm{Gyr}$ is very high and comparable to the age of the Galaxy, raising interesting questions about its progenitor and the initial spin period.

In contrast to LMBPs the evolution of IMBP progenitor systems is not fully understood. Two models for the formation of heavy WDs in close orbits have been proposed: (1) 
the system undergoes a Common Envelope (CE) evolution and spiral-in phase on the Red Giant Branch (RGB) (Tauris 1996) or Asymptotic Giant Branch (AGB) (van den Heuvel 1994), similar to the evolution of high-mass binaries. (2) A massive companion progenitor loses much of its envelope on the late main-sequence or early RGB through highly super-Eddington mass transfer (Tauris et al. 2000; Taam et al. 2000).

For the particular case of PSR J2145-0750, van den Heuvel (1994) proposed an evolutionary channel with a CE spiralin phase on the AGB. In this scenario the progenitor system consisted of a $1-6 M_{\odot}$ donor star, with an orbital period such that this star overflows its Roche lobe on the AGB. During the $\mathrm{CE}$ phase, which is similar to that of high-mass binary pulsar progenitors, the mass accretion onto the pulsar is highly unstable. This results in an incomplete recycling process of the pulsar and can explain the high values for $P$ and $B_{\mathrm{S}}$. One possibility to test these evolutionary channels is to measure the NS and companion masses. Here, we report on new results from timing measurements of the PSR J2145-0750 binary system. They allow us to estimate the inclination and companion mass of the binary. From the detection of a timing parallax we derive an independent distance estimate of the pulsar.

For a pulsar - white dwarf binary system one usually expects that the cooling age of the WD matches the age of the pulsar. This is explained by the fact that the cooling and spindown clocks start ticking at approximately the same time when the companion starts to contract to a WD and the pulsar turns on at the end of the mass transfer phase. The cooling age of the WD companion can be determined from its effective temperature and its mass, using a WD cooling model. We use the parallax distance along with optical observations to revisit the cooling age of the WD companion, based on evolutionary WD cooling models. We discuss the implications of our findings for the evolutionary history of the binary.

\section{Observations}

We have made regular high-precision timing observations of PSR J2145-0750 over a 10 yrs time span using both the 100-m Effelsberg radio-telescope of the Max-Planck-Institut für Radioastronomie in Bonn, Germany, and the 76-m Lovell radio-telescope at Jodrell Bank, UK.

The Effelsberg observations have been carried out since April 1994 at a centre frequency of $1410 \mathrm{MHz}$. Data have been acquired approximately once per month, with a typical observing time of $3 \times 7 \mathrm{~min}$. For the observations we used a 1300-1700 MHz tunable HEMT receiver with a system temperature of $30 \mathrm{~K}$ on the cold sky and an antenna gain of $1.5 \mathrm{~K} \mathrm{Jy}^{-1}$. In order to monitor changes of the dispersion measure (DM) we occasionally collected data at $860 \mathrm{MHz}$, using an uncooled HEMT receiver with a system temperature of $65 \mathrm{~K}$ on the cold sky and a gain of $1.5 \mathrm{~K} \mathrm{Jy}^{-1}$. During the period April 1994-October 1996, the data were obtained using the Effelsberg Pulsar Observation System (EPOS) (Seiradakis et al. 1995; Kramer et al. 1997, 1998). Here, the two circular polarization signals were processed in a $4 \times 60 \times 666 \mathrm{kHz}$ filter bank combined with an incoherent hardware de-disperser. The detected left- and right-hand circular signals in each channel were de-dispersed on-line, added, and folded with the topocentric pulse period to produce a total power pulse profile of $40 \mathrm{MHz}$ bandwidth. Since October 1996 the observations were made with the Effelsberg-Berkeley Pulsar Processor (EBPP), which corrects for the dispersion smearing of the signal using a coherent de-dispersion technique (Hankins \& Rickett 1975). In the total power mode the EBPP provides 32 channels for both polarizations with a maximum total bandwidth of $112 \mathrm{MHz}$, depending on DM and observing frequency (Backer et al. 1997). For PSR J2145-0750 a total bandwidth of $90 \mathrm{MHz}$ was available at $1410 \mathrm{MHz}$. The output signals of each channel were fed into de-disperser boards for coherent on-line de-dispersion and were synchronously folded at the pulse period over 7-min integrations. To obtain a high signal-to-noise ratio polarization profile of PSR J2145-0750 (see Sect. 5.3) we used the EBPP in polarization mode where the four Stokes parameters are available over 32 channels with a total bandwidth of $28 \mathrm{MHz}$.

Lovell observations of PSR J2145-0750 were made from October 1992 to January 1999 at centre frequencies of 606 and $1400 \mathrm{MHz}$. Both circular polarization signals were detected and incoherently de-dispersed in a $2 \times 64 \times 0.125-\mathrm{MHz}$ filter bank at $606 \mathrm{MHz}$ and in a $4 \times 32 \times 1.0-\mathrm{MHz}$ filter bank at $1400 \mathrm{MHz}$. The signals were added and synchronously folded at the pulse period with a typical integration time of 1-3 min. Details of the observing system can be found in Gould \& Lyne (1998).

Both Effelsberg and Lovell data were time stamped with clock information from a local hydrogen maser clock and later synchronized to UTC(NIST) using the signals from the Global Positioning System (GPS). In order to calculate the pulse timeof-arrival (TOA) synthetic templates of the pulse profile were constructed for each backend and observing frequency, and fitted to the observed profiles with template matching procedures (details can be found in Lange et al. 2001). TOA uncertainties were estimated using a method described by Downs \& Reichley (1983). Typical TOA errors for EBPP observations are $2.8 \mu$ s at $860 \mathrm{MHz}$ and $1.9 \mu$ s at $1410 \mathrm{MHz}$, whereas for Lovell observations we found $5.2 \mu \mathrm{s}$ at $606 \mathrm{MHz}$ and $4.5 \mu \mathrm{s}$ at $1400 \mathrm{MHz}$.

\section{Timing analysis}

The combined TOAs, weighted by their individual uncertainties, were fitted to a spin-down model of the pulsar in a binary system with the software package TEMPO ${ }^{1}$, using the DE200 planetary ephemerides (Standish 1990). PSR J2145-0750 lies close to the ecliptic plane, with an ecliptic latitude of only $\beta=5^{\circ} .3$, resulting in less accurate position and proper motion measurements if determined in the equatorial reference frame. In order to minimize covariances between the astrometric parameters we chose ecliptic coordinates for our timing model, performing standard Monte-Carlo simulations to derive reliable values for the proper motion $\mu_{\lambda}$ and $\mu_{\beta}$. Since the pulsar is moving in an almost circular orbit we applied the timing model for binary systems with small eccentricities (ELL1) using the Laplace-Lagrange parameters $\epsilon_{1}$ and $\epsilon_{2}$,

\footnotetext{
${ }^{1}$ http://pulsar.princeton.edu/tempo
} 


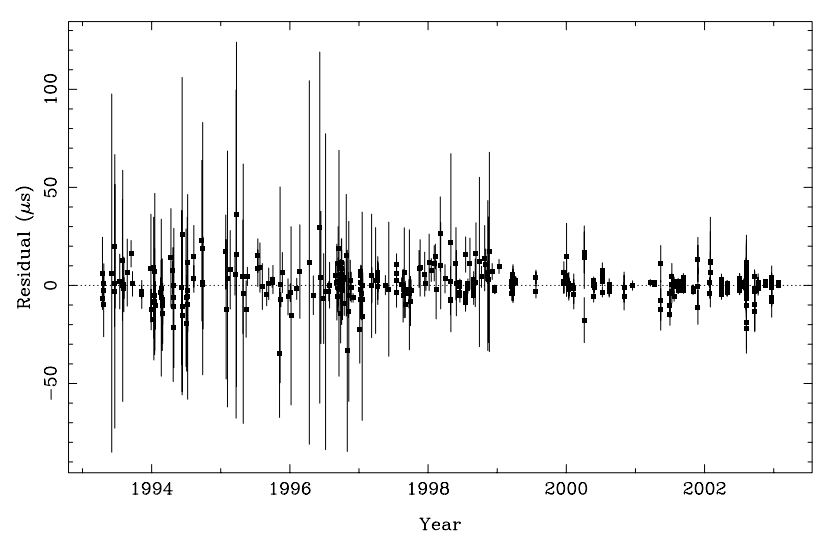

Fig. 1. Post-fit timing residuals of the combined Effelsberg and Lovell $1400 \mathrm{MHz}$ timing data as a function of observing year.

as well as the time of ascending node $T_{\mathrm{ASC}}$ (Lange et al. 2001). The three Keplerian parameters eccentricity $e$, epoch $T_{0}$, and longitude of periastron $\omega$, are then calculated from the former three parameters.

In the fitting procedure the TOA segments obtained with EPOS, EBPP, and Lovell data were fitted for a mutual offset accounting for different templates and TOA reference points in the profiles. Using the full TOA data set at all frequencies we then determined the DM over the 10-yrs period. By excluding all TOAs with lines-of sight to the pulsar lying closer than $30^{\circ}$ to the Sun we analysed the influence of free electrons in the solar system on the DM, but found no variations of the DM or other parameters larger than their $1 \sigma$ uncertainties. However, the multi-frequency timing fit resulted in a detection of a secular DM variation of $\mathrm{d}(\mathrm{DM}) / \mathrm{d} t=-2.2(4) \times 10^{-4} \mathrm{pc} \mathrm{cm}^{-3} \mathrm{yr}^{-1}$. Variations of pulsar DMs have been explained by a simple wedge model of electron density fluctuations in the Galaxy, resulting in a square root dependence of $\mathrm{d}(\mathrm{DM}) / \mathrm{d} t \propto \sqrt{\mathrm{DM}}$ (Backer et al. 1993). Recently, Hobbs et al. (2004) showed that this relation holds for a large group of pulsars, and derived a best-fit of $\mathrm{d}(\mathrm{DM}) / \mathrm{d} t \approx 0.0002 \sqrt{\mathrm{DM}}$, with a scatter of one order of magnitude. For PSR J2145-0750 our measurement of $|\mathrm{d}(\mathrm{DM}) / \mathrm{d} t|$ is consistent with the empirical relation. By holding the values for $\mathrm{DM}$ and $\mathrm{d}(\mathrm{DM}) / \mathrm{d} t$ fixed we then obtained the best-fit timing model for the astrometric, rotational and binary parameters using only the more accurate $1400 \mathrm{MHz}$ TOAs from Effelsberg and Jodrell Bank. We scaled the TOA errors by an appropriate factor to achieve a uniform $\chi^{2} / n_{\text {dof }}=1$ for each TOA segment.

From the best-fit model we obtained post-fit timing residuals that are displayed in Fig. 1. As seen from the figure, we obtained a good coverage of the 10-yrs timing baseline. Note that the EPOS and Lovell TOAs obtained from 1992-1999 have much higher uncertainties than the EBPP data, which is due to the incoherent dispersion removal technique. In order to test the statistical properties of our data we generated a histogram of the deviations of the timing residuals from the model as shown in Fig. 2. The post-fit residuals are consistent with the expected Gaussian distribution. The timing parameters of the best-fit model for PSR J2145-0750 are listed in Table 1. Included in the table are upper limits as well as derived

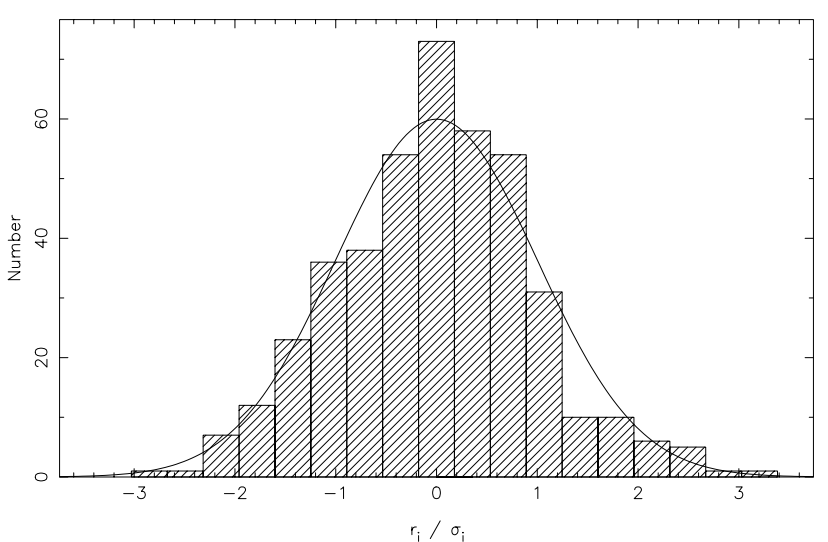

Fig. 2. Distribution of the $1400 \mathrm{MHz}$ post-fit timing residuals normalized by their uncertainties.

parameters. The limits on the pulse frequency second derivative, $\ddot{v}$, and the orbital period derivative, $\dot{P}_{\mathrm{b}}$, were found by allowing the extra parameters to vary one at a time in the global fit. Fitting for the derivatives of both the Laplace-Lagrange parameters, $\dot{\epsilon}_{1}$ and $\dot{\epsilon}_{2}$, led to the upper limits on the eccentricity derivative, $\dot{e}$, and the periastron rate of change, $\dot{\omega}$.

\section{Astrometry and intrinsic pulsar parameters}

\subsection{Parallax, proper motion and transverse velocity}

Pulsar distances are estimated from their DM using models for the electron density distribution in the Galaxy (e.g. Taylor \& Cordes 1993, updated model: Cordes \& Lazio 2002, hereafter NE2001). Independent distance measurements define an absolute distance scale and hence are of fundamental importance to calibrate models like NE2001. For pulsars, distances are derived from trigonometric parallaxes using VLBI observations, or from pulsar timing. In pulsar timing, the parallax is obtained by measuring the time delay of the TOA caused by the curvature of the radio wave fronts. However, the amplitude of the corresponding residual term, $\Delta t_{\pi}(\mu \mathrm{s})=a^{2} \cos \beta /(2 c d)=$ $1.21 \cos \beta / d$, is very small, allowing us to detect a timing parallax only for a very few millisecond pulsars near the ecliptic plane. Here, $a$ is the Earth-Sun distance in kpc, $\beta$ the ecliptic latitude, and $d$ the distance to the pulsar in kpc. Timing parallaxes have been detected for pulsars such as PSR B1855+09 (Kaspi et al. 1991), PSR J1713+0747 (Camilo et al. 1994a), PSR J0437-4715 (Sandhu et al. 1997) and PSR J1744-1134 (Toscano et al. 1999). Nevertheless, due to an ecliptic latitude of only $\beta=5.3$ we were able to detect for the first time a timing parallax for PSR J2145-0750 of $\pi=2.0(6)$ mas. This corresponds to a distance estimate of $d_{\pi}=500_{-120}^{+210} \mathrm{pc}$ and agrees very well with the DM distances, i.e. $d_{\mathrm{DM}}=500 \mathrm{pc}$ from the Taylor \& Cordes (1993) model and $d_{\mathrm{DM}}=570 \mathrm{pc}$ from the NE2001 model (with typical uncertainties of $\sim 10 \%$ ). Using the parallax distance we derive a transverse velocity of $v_{\mathrm{t}}=33(9) \mathrm{km} \mathrm{s}^{-1}$, which is in excellent agreement with scintillation velocities of $31 \pm 25 \mathrm{~km} \mathrm{~s}^{-1}$ obtained by Nicastro \& Johnston (1995) and Johnston et al. (1998).

With a galactic position angle of $289^{\circ}$ the pulsar is moving towards the Galactic plane. This is not in contradiction to the 
Table 1. Timing model for PSR J2145-0750.

\begin{tabular}{|c|c|}
\hline \multicolumn{2}{|l|}{ Measured parameters $^{a}$} \\
\hline Ecliptic longitude, $\lambda$ & $326^{\circ} 02465688(10)$ \\
\hline Ecliptic latitude, $\beta$ & $5.3130779(8)$ \\
\hline Proper motion ${ }^{b}, \mu_{\lambda}\left(\operatorname{mas~yr}^{-1}\right)$ & $-12.37(6)$ \\
\hline Proper motion ${ }^{b}, \mu_{\beta}\left(\right.$ mas yr $\left.^{-1}\right)$ & $-6.8(7)$ \\
\hline Right ascension ${ }^{c}, \alpha(\mathrm{J} 2000)$ & $21^{\mathrm{h}} 45^{\mathrm{m}} 50^{\mathrm{s}} .46726$ \\
\hline Declination $^{c}, \delta(\mathrm{J} 2000)$ & $-07^{\circ} 50^{\prime} 18^{\prime \prime} .375$ \\
\hline Parallax, $\pi$ (mas) & $2.0(6)$ \\
\hline Pulse frequency, $v\left(\mathrm{~s}^{-1}\right)$ & $62.2958888453343(4)$ \\
\hline Pulse frequency derivative, $\dot{v}\left(10^{-16} \mathrm{~s}^{-2}\right)$ & $-1.15481(8)$ \\
\hline Pulse period, $P(\mathrm{~ms})$ & $16.05242365965367(14)$ \\
\hline Period derivative, $\dot{P}\left(10^{-20} \mathrm{~s} \mathrm{~s}^{-1}\right)$ & $2.9757(2)$ \\
\hline Epoch (MJD) & 50800.0 \\
\hline Orbital period, $P_{\mathrm{b}}(\mathrm{d})$ & $6.8389025099(4)$ \\
\hline Projected semi-major axis, $x$ (lt-s) & $10.1641056(6)$ \\
\hline First derivative of $x, \dot{x}\left(10^{-14} 1 \mathrm{t}-\mathrm{s} \mathrm{s}^{-1}\right)$ & $1.8(6)$ \\
\hline$\epsilon_{1}$ & $-0.00000677(8)$ \\
\hline$\epsilon_{2}$ & $-0.00001805(10)$ \\
\hline Epoch of ascending node, $T_{\mathrm{ASC}}(\mathrm{MJD})$ & $50802.29811051(8)$ \\
\hline Eccentricity $^{d}, e$ & $0.00001928(10)$ \\
\hline Epoch of periastron ${ }^{d}, T_{0}(\mathrm{MJD})$ & $50806.108(4)$ \\
\hline Longitude of periastron ${ }^{c}, \omega(\mathrm{deg})$ & $200.5(2)$ \\
\hline Dispersion measure, $\mathrm{DM}\left(\mathrm{pc} \mathrm{cm}^{-3}\right)$ & $9.0031(2)$ \\
\hline Dispersion measure derivative, $\mathrm{d}(\mathrm{DM}) / \mathrm{d} t\left(10^{-4} \mathrm{pc} \mathrm{cm}^{-3} \mathrm{yr}^{-1}\right)$ & $-2.2(4)$ \\
\hline \multicolumn{2}{|l|}{ Measured upper limits ${ }^{e}$} \\
\hline Pulse frequency second derivative, $|\ddot{v}|\left(\mathrm{s}^{-3}\right)$ & $<8 \times 10^{-28}$ \\
\hline Orbital period derivative, $\left|\dot{P}_{\mathrm{b}}\right|\left(\mathrm{s} \mathrm{s}^{-1}\right)$ & $<2.4 \times 10^{-12}$ \\
\hline Eccentricity derivative, $|\dot{e}|\left(\mathrm{s}^{-1}\right)$ & $<2.8 \times 10^{-15}$ \\
\hline Periastron rate of change, $|\dot{\omega}|\left(\operatorname{deg~yr}^{-1}\right)$ & $<0.47$ \\
\hline \multicolumn{2}{|l|}{ Derived parameters } \\
\hline Galactic longitude, $l$ & $47^{\circ} .78$ \\
\hline Galactic latitude, $b$ & $-42^{\circ} .08$ \\
\hline Parallax distance $(\mathrm{pc})$ & $500_{-120}^{+210}$ \\
\hline Composite proper motion, $\mu$ (mas $\mathrm{yr}^{-1}$ ) & 14.1(4) \\
\hline Transverse velocity, $v_{\mathrm{t}}\left(\mathrm{km} \mathrm{s}^{-1}\right)$ & $33(9)$ \\
\hline Mass function, $f_{\mathrm{m}}\left(M_{\odot}\right)$ & $0.024105570(4)$ \\
\hline Pulsar characteristic age (Gyr) & $10.4(5)$ \\
\hline Pulsar surface magnetic field $\left(10^{8} \mathrm{G}\right)$ & $6.35(14)$ \\
\hline Timing RMS ( $\mu \mathrm{s})$ & 2.7 \\
\hline
\end{tabular}

${ }^{a}$ Uncertainties quoted are in the last digit(s) and represent $2 \sigma$ estimates (twice the formal TEMPO errors).

${ }^{b}$ Calculated from Monte-Carlo simulations.

${ }^{c}$ Calculated from ecliptic coordinates.

${ }^{d}$ Calculated from the ELL1 model.

${ }^{e}$ Upper limits represent $95 \%$ C.L.

idea that pulsars are born in the Galactic plane and then move to higher latitudes, as the MSP population is very old ( $\sim 1 \mathrm{Gyr})$ and has already reached a dynamic equilibrium in the Galactic gravitational potential (e.g. Toscano et al. 1999). In this scenario PSR J2145-0750, with a characteristic age of $10 \mathrm{Gyr}$ (see Sect. 4.3), would have already performed many oscillations in the Galactic potential described by Kuijken \& Gilmore (1989).

\subsection{Doppler corrections}

Accelerations in the Galactic gravitational potential introduce contributions to the observed period derivative $\dot{P}$ which cannot be neglected for MSPs that have spin-down rates of about six orders of magnitude lower than those of normal pulsars. Doppler effects arise due to (a) the Galactic differential 
rotation, (b) the vertical acceleration in the Galaxy (Damour \& Taylor 1991), and (c) the transverse velocity of the pulsar (Shklovskii 1970). Following these authors, the Doppler correction to the period derivative can be written as

$\left(\frac{\dot{P}}{P}\right)^{\mathrm{D}}=\frac{a_{z} \sin b}{c}-\frac{v_{0}^{2}}{c R_{0}} \cos b\left(\cos l+\frac{\beta}{\sin ^{2} l+\beta^{2}}\right)+\frac{\mu^{2} d}{c}$,

where $R_{0}$ and $v_{0}$ denote the galactocentric radius and galactic circular velocity of the Sun, respectively, $l$ and $b$ the galactic longitude and latitude of the pulsar, and $\beta=d / R_{0}-\cos l$. We choose $R_{0}=8.0 \mathrm{kpc}$ and $v_{0}=220 \mathrm{~km} \mathrm{~s}^{-1}$ (Reid 1993). $a_{z}$ is the vertical component of the galactic acceleration and can be calculated from the Kuijken \& Gilmore (1989) model of the Galactic potential. For PSR J2145-0750 we derive a kinematic correction of $(\dot{P} / P)^{\mathrm{D}}=3.3(7) \times 10^{-19} \mathrm{~s}^{-1}$, where the major contribution $(73 \%)$ comes from the Shklovskii term $\left(\mu^{2} d\right) / c$.

\subsection{Characteristic age and magnetic field}

Subtracting the kinematic contributions from the observed spin-down rate of PSR J2145-0750 we obtain an intrinsic spindown rate of $\dot{P}^{\text {intr }}=\dot{P}-\dot{P}^{\mathrm{D}}=2.45(11) \times 10^{-20} \mathrm{~s} \mathrm{~s}^{-1}$ which is $18 \%$ smaller than the observed value.

It is usually assumed that the evolution of the spin frequency $v=1 / P$ can be described by a power-law $\dot{v} \propto-v^{n}$, where $n$ is the so-called braking index. Then the pulsar age can be calculated from

$\tau=\frac{P}{(n-1) \dot{P}}\left[1-\left(\frac{P_{0}}{P}\right)^{n-1}\right]$,

where $P_{0}$ is the initial period of the pulsar. Under the assumptions that $P_{0} \ll P$ and that the spin-down is due to magnetic dipole braking $(n=3)$, the spin-down age is given by $\tau_{\mathrm{c}}=P /(2 \dot{P})$, the characteristic age of the pulsar. Subtracting the Doppler contributions, for PSR J2145-0750 we thus obtain $\tau_{\mathrm{c}}=P /\left(2 \dot{P}^{\text {intr }}\right)=10.4(5)$ Gyr. As the uncertainty of $\tau_{\mathrm{c}}$ caused by the a priori unknown $n$ and $P_{0}$ can be considerable, an independent age estimate, as given by the cooling age of the pulsar companion (see Sect. 6), is highly desirable.

Assuming a pure dipolar magnetic field and using the intrinsic spin-down rate we can estimate the surface magnetic field of the pulsar to be $B_{\mathrm{S}}=3.2 \times$ $10^{19} \sqrt{P \dot{P}^{\text {intr }}} \mathrm{G}=6.35(14) \times 10^{8} \mathrm{G}$. This magnetic field is more than three orders of magnitude smaller than for normal pulsars, a clear indication than PSR J2145-0750 has gone through a recycling phase.

\subsection{Timing instabilities}

Any remaining non-gaussian noise in timing residuals is generally explained by rotational instabilities intrinsic to the pulsar, or by an incomplete timing model, such as unmodelled DM variations or the existence of a planet in the system. For PSR J2145-0750 a fit for $\ddot{v}$ over the full timing baseline resulted in $\ddot{v}=-5(3) \times 10^{-28} \mathrm{~s}^{-3}$. As this measurement is hardly significant we conservatively quote $\ddot{v}$ as an upper limit in Table 1. At the same time, we do not detect any DM variations that are not accounted for in the timing model. A simultaneous fit of our multi-frequency data for third- and fourth-order derivatives of DM as well as for $\ddot{v}$ resulted in a similar $\ddot{v}$ value, while $\mathrm{d}^{2}(\mathrm{DM}) / \mathrm{d} t^{2}$ and $\mathrm{d}^{3}(\mathrm{DM}) / \mathrm{d} t^{3}$ were not significant.

Arzoumanian et al. (1994) analysed the timing noise of a number of slow and millisecond pulsars by quantifying the noisiness with the noise parameter $\Delta_{8}=\log \left(|\ddot{v}| t^{3} /(6 v)\right)$, where $t=10^{8} \mathrm{~s}$. They quote a fit-by-eye to the data of the form $\Delta_{8}=6.6+0.6 \log \dot{P}$, with a large scatter of the data points. Lommen (2002) revised this relation using new MSP data and showed that at least seven MSPs have significantly lower $\Delta_{8}$ 's than presented by Arzoumanian et al. (1994).

In order to estimate the timing noise of PSR J2145-0750 we divided the total data set into three sub-intervals, each spanning $10^{8} \mathrm{~s} \approx 3.2 \mathrm{yr}$ of TOAs. For each sub-interval we transformed epoch, pulse frequency $v$, and epoch of ascending node $T_{\text {asc }}$ to an epoch near the centre of each sub-interval. We then fitted the timing model of the pulsar, holding DM and $\mathrm{d}(\mathrm{DM}) / \mathrm{d} t$ fixed and allowing $\ddot{v}$ to vary. The resulting $\Delta_{8}$ 's for each sub-interval are $-4.0,-4.1$, and -4.3 , i.e. slightly larger than the predicted value of $\Delta_{8}=-5.1$. We thus conclude that PSR J2145-0750 is a possible candidate for intrinsic timing noise. Future timing observations will resolve this assertion.

\subsection{Secular changes in orbital parameters}

For the first time we detected a secular variation of the projected semi-major axis of PSR J2145-0750 of $\dot{x}=1.8(6) \times$ $10^{-14} \mathrm{lt}-\mathrm{s} \mathrm{s}^{-1}$. In order to test the robustness of this measurement we divided the total set into five sub-intervals, each spanning two years of TOAs. For every sub-interval the TOAs were fitted to the timing model of the pulsar, with the variation of $x$ held fixed at zero. Hereby we transformed epoch, pulse frequency $v$, and epoch of ascending node $T_{\text {asc }}$ to an epoch near the centre of each sub-interval. The fits resulted in an increase of $x$ with time, with a slope of $1.7(9) \times 10^{-14} \mathrm{lt}_{-} \mathrm{s} \mathrm{s}^{-1}$, confirming the value from the global fit.

The timing data showed no evidence for secular changes in other orbital parameters. In principle, the binary system could undergo both a general relativistic (GR) advance of periastron and an orbital decay due to the emission of gravitational radiation (Damour \& Taylor 1991), i.e.

$$
\begin{aligned}
\dot{\omega}^{\mathrm{GR}}= & 3\left(\frac{P_{\mathrm{b}}}{2 \pi}\right)^{-5 / 3} \frac{1}{1-e^{2}}\left(T_{\odot} M\right)^{2 / 3} \\
\dot{P}_{\mathrm{b}}^{\mathrm{GR}}= & -\frac{192 \pi}{5}\left(\frac{P_{\mathrm{b}}}{2 \pi}\right)^{-5 / 3} \frac{\left(1+\frac{73}{24} e^{2}+\frac{37}{96} e^{4}\right)}{\left(1-e^{2}\right)^{7 / 2}} \\
& \times T_{\odot}^{5 / 3} m_{1} m_{2} M^{-1 / 3}
\end{aligned}
$$

where the masses are in Solar units and $T_{\odot}=\left(G M_{\odot} / c^{3}\right)$.

If we assume a pulsar mass of $m_{1}=1.4 M_{\odot}$ and a companion mass of $m_{2}=0.51 M_{\odot}$ (using the mass function and a median inclination $\langle i\rangle=60^{\circ}$ ), we expect $\dot{\omega}^{\mathrm{GR}}=0.0124 \mathrm{yr}^{-1}$ and $\dot{P}_{\mathrm{b}}^{\mathrm{GR}} \sim-5.1 \times 10^{-16} \mathrm{~s} \mathrm{~s}^{-1}$. However, as PSR J2145-0750 has a very small eccentricity, all effects of secular variations in $\omega$ are fully absorbed by the redefinition of the binary period and are therefore not observable in this system. The observed 
upper limit for $\dot{P}_{\mathrm{b}}$ is four orders of magnitude higher which makes any detection of the GR orbital decay highly unlikely.

Moreover, the Shklovskii effect discussed in Sect. 4.2 also affects the observed value of $\dot{P}_{\mathrm{b}}$. Using the results from Sect. 4.2, we expect a contribution of $\dot{P}_{\mathrm{b}}^{\text {Shk }}=2.4(5) \times 10^{-19} \mathrm{~s}^{-1} \times$ $P_{\mathrm{b}}=1.4(3) \times 10^{-13} \mathrm{~s} \mathrm{~s}^{-1}$. This value is not only of different sign as the expected GR contribution but also about three orders of magnitude larger. Nevertheless, the Shklovskii term is still consistent with the observations as it is yet a factor of 20 smaller than our derived upper limited.

\section{Orbital inclination and mass}

\subsection{Secular change of $x$}

The observed secular change of the projected semi-major axis $x=a_{\mathrm{p}} \sin i / c$ could, in principle, result from a variation of the major axis of the pulsar orbit, $a_{\mathrm{p}}$, of the orbital inclination, $i$, or a combination of both. The contributions to $\dot{x}$ are (Damour \& Taylor 1992; Kopeikin 1996):

$\left(\frac{\dot{x}}{x}\right)^{\mathrm{obs}}=\left(\frac{\dot{a}_{\mathrm{p}}}{a_{\mathrm{p}}}\right)^{\mathrm{GW}}+\left(\frac{\dot{x}}{x}\right)^{\mathrm{D}}+\left(\frac{\dot{x}}{x}\right)^{\mathrm{PM}}$.

The emission of gravitational waves $(\mathrm{GW})$ leads to a shrinking of the pulsar orbit given by $\left(\dot{a}_{\mathrm{p}} / a_{\mathrm{p}}\right)^{\mathrm{GW}}=2 / 3 \times\left(\dot{P}_{\mathrm{b}} / P_{\mathrm{b}}\right)^{\mathrm{GW}}($ e.g. Doroshenko et al. 2001). With Eq. (4) one expects $\left|\dot{a}_{\mathrm{p}} / a_{\mathrm{p}}\right|^{\mathrm{GW}} \sim$ $5.8 \times 10^{-22} \mathrm{~s}^{-1}$ which is seven orders of magnitude smaller than the observed $|\dot{x} / x|^{\text {obs }}=1.8 \times 10^{-15} \mathrm{~s}^{-1}$. In general, $\left|\dot{a}_{\mathrm{p}} / a_{\mathrm{p}}\right|$ will be of the same order of magnitude as $\left|\dot{P}_{\mathrm{b}} / P_{\mathrm{b}}\right|$ for typical astrophysical processes in a binary. With the observed upper limit of $\dot{P}_{\mathrm{b}}<2.4 \times 10^{-12} \mathrm{~s}^{-1}$ we derive $\left|\dot{P}_{\mathrm{b}} / P_{\mathrm{b}}\right|<4.1 \times 10^{-18} \mathrm{~s}^{-1}$, still three orders of magnitude smaller than $|\dot{x} / x|^{\text {obs }}$. Therefore, the nonzero $\dot{x}$ is most likely not caused by orbital evolution.

The Doppler correction affects the light-travel time across the orbit in the same way as the pulse period, so that the term $(\dot{x} / x)^{\mathrm{D}}$ is identical to the one for the period derivative (Eq. (1), see Damour \& Taylor 1992). Its value is therefore four orders of magnitude smaller than the observed value $(\dot{x} / x)^{\text {obs }}$.

We conclude that the nonzero $\dot{x}$ must arise from a change in the observed inclination $i$ of the orbit. The relative motion of the binary and the observer causes a change of the orientation of the line-of-sight to the binary which results in an apparent change of $i$. Kopeikin (1996) derived

$$
\left(\frac{\dot{x}}{x}\right)^{\mathrm{PM}}=\mu \cot i \sin \theta
$$

where $\theta$ denotes the difference of the position angle of proper motion and the position angle of ascending node. As $\theta$ is a priori unknown, we can only derive a firm upper limit for $i$ using $|\sin \theta|<1$ and the lower limit of allowed values for $\dot{x}$ from Table 1, i.e. $i<\tan ^{-1}\left(\mu x / \dot{x}_{\min }\right)=61^{\circ}$.

We performed Monte-Carlo simulations to derive the distribution of inclination angles within this constraint, following a procedure described by Nice et al. (2001). Assuming that the orientation of the binary in space is arbitrary, $\theta$ is a uniformly distributed random variable and $i$ is a random variable distributed with uniform probability in $\cos i$. From these distributions we select values of $\theta$ and $i$ and retain only those combinations which satisfy Eq. (6) within the measurement errors.

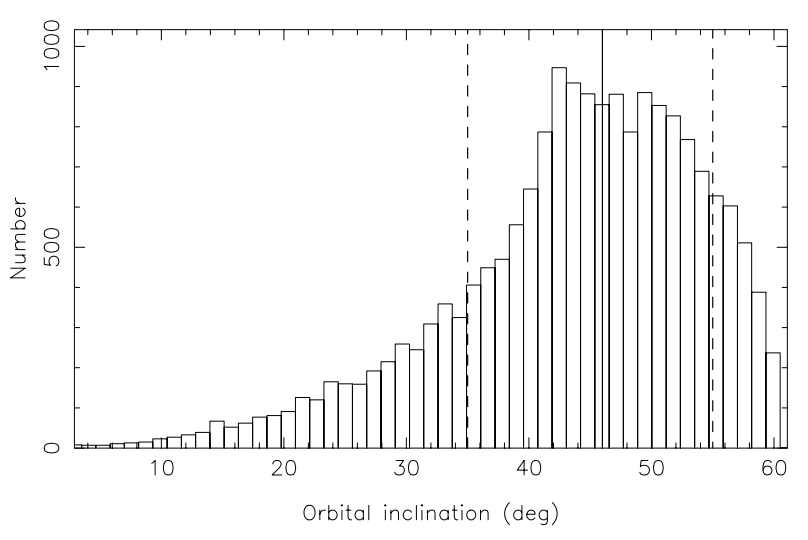

Fig. 3. Distribution of orbital inclinations $i$ resulting from MonteCarlo simulations (see Sect. 5.1). The median of the distribution is $46^{\circ}$ (solid line), and its lower and upper $1 \sigma$ values are at $35^{\circ}$ and $55^{\circ}$, respectively (dashed lines).

The resulting distribution for $i$ is presented in Fig. 3, showing a broad peak towards higher inclinations. The median value and its asymmetric errors are $i=46^{\circ}{ }_{-11}^{\circ}$ ( $68 \%$ C.L.).

\subsection{Non-detection of Shapiro delay}

The propagation of radio signals from pulsars in binary systems is affected by a general-relativistic time delay of the signals in the gravitational field of the companion star. For a pulsar in a circular orbit this "Shapiro delay" (Shapiro 1964) is given by

$\Delta t=-2 m_{2} T_{\odot} \ln \left[1-\sin i \sin \left(\Phi-\Phi_{0}\right)\right]$,

where $\Phi$ is the orbital phase in radians, $\Phi_{0}$ is the phase of the ascending node, and $T_{\odot}=\left(G M_{\odot} / c^{3}\right)$. In practice, Shapiro delay is conveniently expressed in terms of two observables, the "range" $r=m_{2} T_{\odot}$ and the "shape" $s=\sin i$ (Ryba \& Taylor 1991), the post-Keplerian orbital parameters which allow a determination of the companion mass, $m_{2}$, and the orbital inclination, $i$. However, for small inclination angles the variation of $\Delta t$ over the orbit is nearly sinusoidal and cannot be separated from a small variation in $x$. For edge-on orbits $\left(i \approx 90^{\circ}\right), \Delta t$ peaks at $\Phi-\Phi_{0}=\pi / 2$, where the pulsar is behind the companion. Here, the covariance with the Keplerian parameters breaks, and a measurement of Shapiro delay (and hence $r$ and $s$ ) is possible. Because high inclination orbits are relatively rare, Shapiro delay has been detected in only five pulsar-white dwarf binaries, PSR B1855+09 (Ryba \& Taylor 1991), PSR J1713+0747 (Camilo et al. 1994a), PSR J0437-4715 (van Straten et al. 2001), PSR J1909-3744 (Jacoby et al. 2003), PSR J1640+2224 (Löhmer et al. 2004), and perhaps in PSR J0751+1807 (Nice et al. 2003).

We did not detect Shapiro delay in the timing data of PSR J2145-0750. However, we can use the non-detection to place limits on allowed values for $i$ and $m_{2}$. Following a procedure described by Nice et al. (2001), we tested a 2D grid of timing models, that include the Shapiro parameters in the range of $0 \leq \sin i \leq 1$ and $0 \leq m_{2} \leq 1.4 M_{\odot}$, allowing all other parameters to vary. The resulting $\chi^{2}$ values are mostly very similar to a model without Shapiro delay. However, for models with 


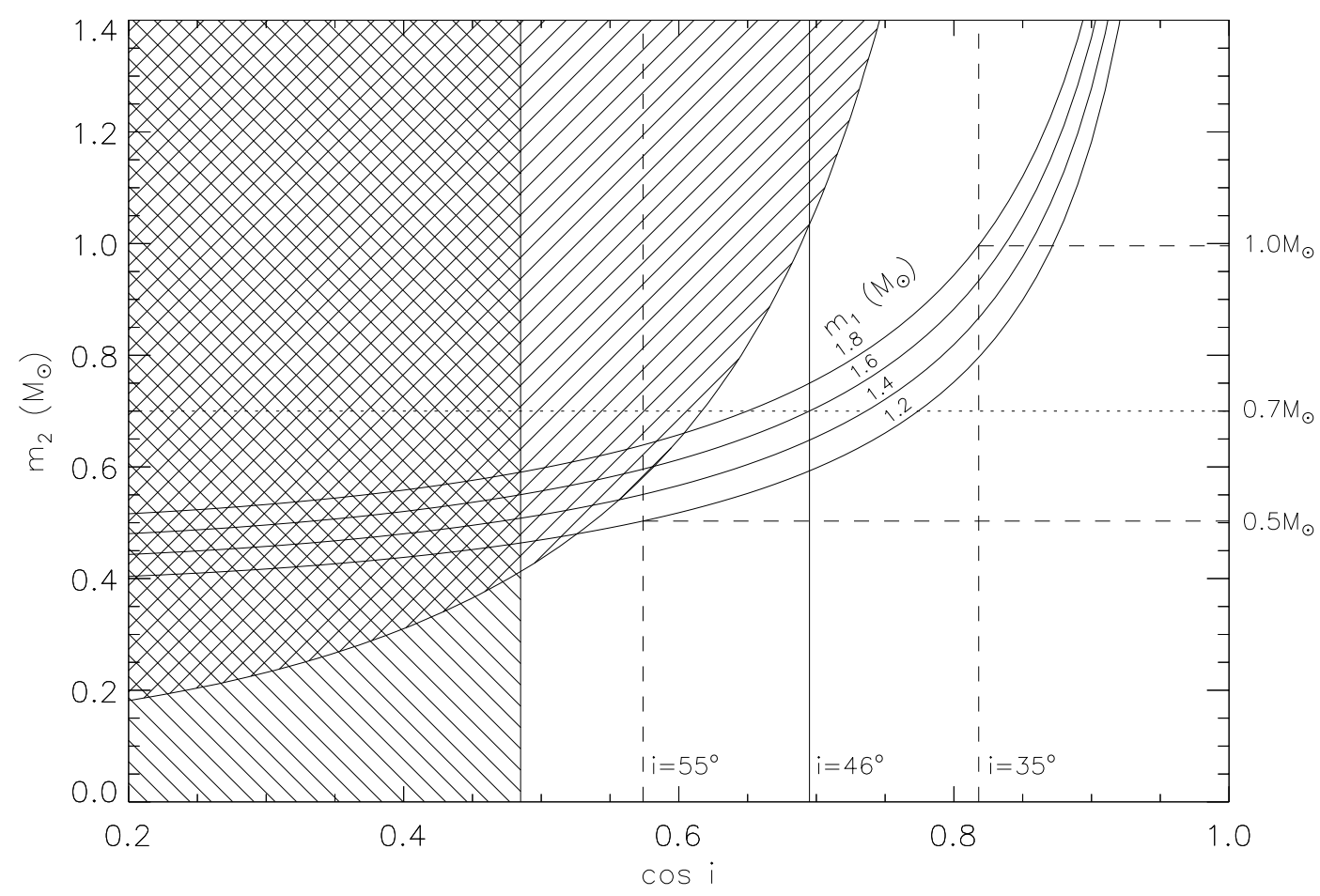

Fig. 4. Constraints on orbital inclination, $i$, and WD mass, $m_{2}$, from $\dot{x}$-measurement and limits on Shapiro delay. Points inside the hatched rectangle are excluded by measured $\dot{x}$, points inside the curved hatched area are excluded by the non-detection of Shapiro delay. From the mass function we calculated the contours for constant neutron star masses, indicated by the solid curves. The vertical lines denote the median value ( $i=46^{\circ}$, solid) and its $1 \sigma$ errors (at $35^{\circ}$ and $55^{\circ}$, dashed) for the distribution of inclination angles derived from the observed $\dot{x}$. From these uncertainties we derive the companion mass limits $0.5 M_{\odot} \leq m_{2} \leq 1.0 M_{\odot}$ displayed by the horizontal dashed lines. Photometric studies of the WD companion (see Sect. 6) result in a lower mass limit of $0.7 M_{\odot}$ (horizontal dotted line).

high inclination angles and/or high companion masses we obtain significantly higher values for $\chi^{2}$. The failure of these models means that one would have detected Shapiro delay had these been the true values of $i$ and $m_{2}$. In Fig. 4, the parameter space excluded by models with $\Delta \chi^{2}>9$ (i.e., $3 \sigma$ ) is shown.

\subsection{Polarimetry}

During the recycling process of MSPs the neutron star is spunup by mass transfer from the companion star which is expected to cause an alignment of pulsar spin and orbital angular momentum of the binary system (Phinney \& Kulkarni 1994). Assuming that this holds for PSR J2145-0750 it is then possible to constrain the orbital inclination from the known geometry of the pulsar magnetosphere. We therefore observed PSR J2145-0750 at $1410 \mathrm{MHz}$ using the EBPP in polarization mode and derived a position angle that is similar to the findings of Sallmen (1998) and Stairs et al. (1999). The profile of PSR J2145-0750 shows at least three components, with a bridge of emission between the two strongest components. Unfortunately, the linear intensity at $1410 \mathrm{MHz}$ is very weak (but see also Xilouris et al. 1998; Sallmen 1998), and the accompanying position-angle swing is extremely complex, with multiple orthogonal mode changes (Stairs et al. 1999). Applying a rotating vector model (RVM) (Radhakrishnan \& Cooke 1969) to the observed position angle usually leads to high uncertainties in the RVM fit. We fitted a RVM curve to the position angle of the whole profile (including the precursor) and found a best fit for a magnetic inclination of $\alpha \sim 52^{\circ}$ and an impact angle of $\sigma \sim-3^{\circ}$. These values are considerably different from those found by Sallmen (1998) which is probably due to the inclusion of the precursor in our fit leading to a longer baseline in pulse longitude. Even though the position angle is poorly constrained leading to considerable errors in the RVM fits, when we apply our best-fit values the angle between the pulsar rotation axis and the line-of-sight results in $\alpha+\sigma \sim 49^{\circ}$. If one assumes that the pulsar rotation axis is aligned with the orbital momentum, one derives $i=\alpha+\sigma$ (or $\left.i=180^{\circ}-(\alpha+\sigma)\right)$, i.e. $i \sim 49^{\circ}$ (or $\left.i \sim 131^{\circ}\right)$.

One generally needs to be careful in interpreting the position angles observed in recycled pulsars as being the reflection of the underlying geometry. The position angle swings are typically much flatter and often do not resemble the expected S-like swing (e.g. Xilouris et al. 1998; Stairs et al. 1999). Given this caveat, it is interesting to note that the fitted inclination angle agrees very well with the median value of $i=46^{\circ} \mathrm{ob}$ tained from our $\dot{x}$ measurement. We conclude that the analysis of the pulsar magnetosphere by polarimetry results in a consistent picture of the orbital inclination, strongly supporting our earlier findings.

\subsection{Companion mass}

As demonstrated, the $\dot{x}$ measurement and the limits on Shapiro delay for PSR J2145-0750 can be used to put constraints on the orbital inclination, $i$, and the companion mass, $m_{2}$, as shown in 
Fig. 4. From the observed $\dot{x}$ we derive an upper limit on the inclination angle of $i<61^{\circ}$, so that all points with $\cos i<0.48$ are excluded as indicated by the hatched rectangle in the figure. The curved hatched area is the parameter space excluded due to the non-detection of Shapiro delay in the binary. From the mass function of PSR J2145-0750 we derive the displayed contours for constant neutron star masses.

We cannot put any constraints on the companion mass from evolutionary considerations of the binary as the PSR J2145-0750 system most probably went through a phase of highly unstable mass accretion (van den Heuvel 1994). Thus, a unique relation between the orbital period, $P_{\mathrm{b}}$, and the secondary mass, $m_{2}$, as found for LMBPs, is not applicable for this system. However, from the distribution of allowed inclination angles (see Sect. 5.1), and the contours for constant pulsar masses we can estimate a range of allowed companion masses. Using recent results of neutron star mass measurements (Thorsett \& Chakrabarty 1999; Stairs 2004, and references therein) we apply a range of $1.2 M_{\odot} \leq m_{1} \leq 1.8 M_{\odot}$ for the pulsar mass (for a discussion see Sect. 7). As shown in Fig. 4, for inclination angles within their $1 \sigma$ uncertainties we derive a range of allowed companion masses of $0.5 M_{\odot} \leq m_{2} \leq$ $1.0 M_{\odot}$.

\section{The nature and cooling age of the white dwarf companion}

From the timing analysis we derive a range of companion masses of $0.5 M_{\odot} \leq m_{2} \leq 1.0 M_{\odot}$, and a global minimum of $m_{2, \min }=0.48 M_{\odot}$ for an extremely low pulsar mass of $m_{1}=1.2 M_{\odot}$ (see Sect. 7). The upper mass limit for a helium (He)-core WD with solar metallicity is $M_{\mathrm{He}-\mathrm{WD} \text {, } \max } \approx 0.45 M_{\odot}$. Hence we conclude that the companion is a WD with carbon/oxygen $(\mathrm{CO})$ core in the intermediate WD mass range. This result is consistent with a CE evolution of the binary system as proposed by van den Heuvel (1994). Using the derived mass range of the WD companion and its photometrically determined effective temperature it is possible to determine an age of the binary system. This age is based on WD cooling models coming from stellar evolution theory and can be compared with the characteristic pulsar age.

Optical observations of the WD companion with the HST from Lundgren et al. (1996) yielded $m_{V}=23.7 \pm 0.1$ and $m_{I}=23.0 \pm 0.1$. From these measurements the authors derived an effective temperature of $T_{\text {eff }}=5800 \pm 300 \mathrm{~K}$ using the color-temperature calibration from Bergeron et al. (1995) and the bolometric correction from Monet et al. (1992). With the same observational data from Lundgren et al. (1996), Hansen $\&$ Phinney (1998) estimated $T_{\text {eff }}=6235 \pm 970 \mathrm{~K}$ based on the $V-I$ broad-band colors of Bergeron et al. (1995). As conservative limits, we thus use a range of effective temperatures for the companion between 5250 and $7200 \mathrm{~K}$.

Figure 5 shows an age $-T_{\text {eff }}$ diagram with evolutionary tracks of CO-core WD models from Blöcker (1995) and Blöcker et al. (1997). The horizontal lines indicate the observational range for $T_{\text {eff }}$. The curve at the very left of the diagram belongs to the CO-core WD model with the lowest mass, followed by curves of increasing core mass while going to the

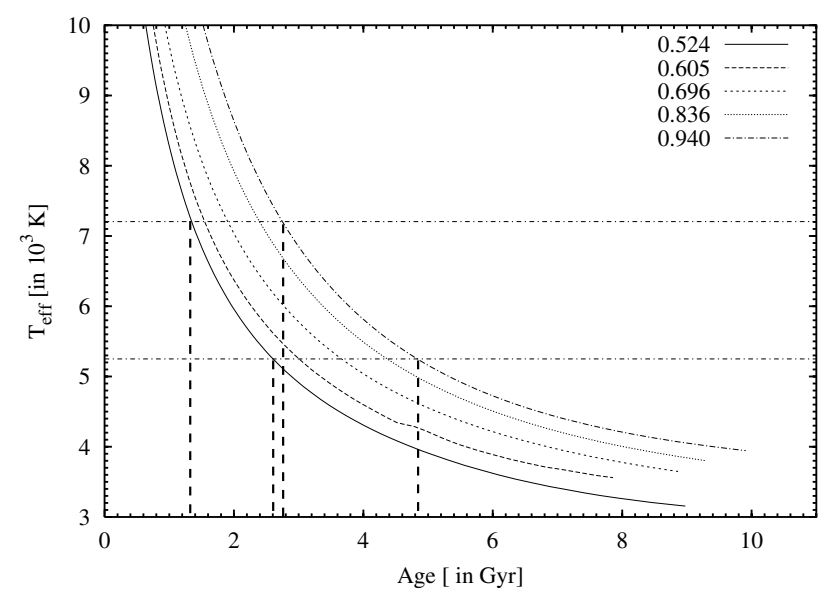

Fig. 5. Age-temperature diagram with cooling curves from evolutionary models of Blöcker (1995) and Blöcker et al. (1997) for CO-core WDs $\left(0.52 M_{\odot}<M_{\mathrm{WD}}<0.94 M_{\odot}\right)$. The labels give the WD masses in $M_{\odot}$, and the straight horizontal lines indicate the possible range of effective temperatures for the WD companion of PSR J2145-0750 according to the studies of Lundgren et al. (1996) and Hansen \& Phinney (1998), i.e. $5250 \mathrm{~K} \leq T_{\text {eff }} \leq 7200 \mathrm{~K}$. The thick dashed vertical lines mark the intersections of these lines with the cooling curves from the least and the most massive CO-core model $\left(M_{\mathrm{WD}}=0.524\right.$ and $0.940 M_{\odot}$, respectively) to indicate the corresponding age range.

right. The WD models with masses of 0.524 and $0.940 M_{\odot}$ roughly bracket the mass range for the companion derived from the timing analysis. As the vertical dashed lines in Fig. 5 illustrate, a WD with $m_{\mathrm{WD}}=0.524 M_{\odot}$ would have an age of $1.3 \ldots 2.6 \mathrm{Gyr}$, whereas a massive WD with $m_{\mathrm{WD}}=0.940 M_{\odot}$ would have an age of $2.8 \ldots 4.9 \mathrm{Gyr}$.

Using the photometric measurements of Lundgren et al. (1996) in the $B, V$, and $I$ bands together with our parallax distance for PSR J2145-0750, the mass, age, and effective temperature of the WD companion can be further constrained. With $d=500_{-120}^{+210} \mathrm{pc}$ and the apparent magnitudes $m_{B}=23.9$, $m_{V}=23.7$, and $m_{I}=23.0$ from Lundgren et al. (1996) we find absolute magnitudes $M_{B}=15.4_{-0.8}^{+0.6}, M_{V}=15.2_{-0.8}^{+0.6}$, and $M_{I}=14.5_{-0.8}^{+0.6}$. Applying the bolometric corrections $B C_{I}=0.5$ from Monet et al. (1992) and $B C_{V}=-0.2$ from Bergeron et al. (1995), from both $M_{V}$ and $M_{I}$ we obtain a bolometric brightness of $M_{\mathrm{bol}}=15.0_{-0.8}^{+0.6}$, which finally gives a WD luminosity of $\log \left(L / L_{\odot}\right)=-4.1_{-0.2}^{+0.3}$. This value can be compared with the luminosity predictions from the WD cooling models from Blöcker (1995) and Blöcker et al. (1997) for $T_{\text {eff }}=5250 \ldots 7200 \mathrm{~K}$. The model parameters are summarized in Table 2.

As can be seen from Table 2, using $\log \left(L / L_{\odot}\right)=-4.1$ as an additional constraint a CO-core WD with $m_{2} \lesssim 0.7 M_{\odot}$ can be ruled out as companion for PSR J2145-0750, since WDs with lower masses would be too luminous. Here, a WD with $m_{2} \approx$ $0.7 M_{\odot}$ would just have $\log \left(L / L_{\odot}\right)=-4.1$ in accordance with the observations, and the cooling age would be $\tau_{\text {cool }}=3.7 \mathrm{Gyr}$. On the other hand, a WD with $m_{2}=0.94 M_{\odot}$ would be too faint at a temperature of $T_{\text {eff }}=5250 \mathrm{~K}$. Using $\log \left(L / L_{\odot}\right)=-4.1$ as constraint, we obtain $\tau_{\text {cool }}=3.4 \mathrm{Gyr}$ from the $0.94 M_{\odot}$ cooling model, which in turn leads to a new upper limit for the effective temperature of $T_{\text {eff }}=6400 \mathrm{~K}$ (see Fig. 5). Therefore, from 
Table 2. Cooling properties of the CO-core WD companion of PSR J2145-0750. All values are taken from WD evolutionary models of Blöcker (1995) and Blöcker et al. (1997).

\begin{tabular}{llll}
\hline \hline \multicolumn{4}{c}{ Model parameters of the WD companion } \\
\hline$M_{\mathrm{WD}} / M_{\odot}$ & $\log \left(L / L_{\odot}\right)$ & $T_{\text {eff }} / \mathrm{K}$ & $\tau_{\text {cool }} / \mathrm{Gyr}$ \\
\hline 0.524 & -3.348 & 7200 & 1.35 \\
0.524 & -3.913 & 5250 & 2.60 \\
\hline 0.605 & -3.448 & 7200 & 1.50 \\
0.605 & -3.995 & 5250 & 3.00 \\
\hline 0.696 & -3.532 & 7200 & 1.90 \\
0.696 & -4.087 & 5250 & 3.70 \\
\hline 0.836 & -3.661 & 7200 & 2.40 \\
0.836 & -4.285 & 5250 & 3.40 \\
\hline 0.940 & -3.825 & 7200 & 2.80 \\
0.940 & -4.850 & 5250 & 4.85 \\
\hline
\end{tabular}

the overall comparison of observational constraints and WD models, we conclude that the PSR J2145-0750 companion has a mass of $0.7 M_{\odot} \leq m_{2} \leq 1.0 M_{\odot}$, an effective temperature of $T_{\text {eff }}=5750 \pm 600 \mathrm{~K}$, and a cooling age of $\tau_{\text {cool }}=3.6(2)$ Gyr.

The WD mass range derived here is in accordance with the results from our timing analysis (see Sect. 5.4), but provides a tighter lower limit of $0.7 M_{\odot}$. On the other hand, the results on the WD properties are also in agreement with previous studies from Lundgren et al. (1996) and Hansen \& Phinney (1998). Taking the mass range $0.7 M_{\odot} \leq m_{2} \leq 0.9 M_{\odot}$, we can also compare our results with those obtained from the recent WD models of Richer et al. (2000). Using their models, we obtain $T_{\text {eff }}=5700 \pm 350 \mathrm{~K}$ and $\tau_{\text {cool }}=4.4(1) \mathrm{Gyr}$ in line with our results.

\section{Discussion and conclusions}

Our timing observations of the PSR J2145-0750 binary system constrain the orbital inclination angle to be $i<61^{\circ}$, with a median value of $i=46^{\circ}$. Polarimetric studies of the pulsar magnetosphere lead to a consistent result but cannot provide stronger constraints on the orbital inclination. From a statistical analysis of allowed inclination angles we are able to derive proper mass limits for the pulsar companion if we apply a realistic estimate for the pulsar mass. Neutron stars are believed to have masses close to the Chandrasekhar value of $\sim 1.35 M_{\odot}$. Thorsett \& Chakrabarty (1999) reviewed all neutron star mass measurements and found a remarkably narrow distribution of $1.35(4) M_{\odot}$. Recent observations revealed neutron stars that have lower masses, e.g. PSR J0737-3039B with $1.250(5) M_{\odot}$ in the newly detected double-pulsar system (Lyne et al. 2004), and PSR J1141-6545 with 1.30(2) $M_{\odot}$ in a NS-WD system (Bailes et al. 2003). New timing observations showed that recycled pulsars in NS-WD binaries tend to have masses that are significantly higher than the canonical value of $1.35 M_{\odot}$ (Nice 2003), which is generally explained by a long phase of extended mass transfer from the companion to the NS. This also holds true for PSR J0621+1002 with a pulsar mass of $\sim 1.7 M_{\odot}$ (Splaver et al. 2002), an IMBP system with properties similar to PSR J2145-0750. The currently known NS masses in recycled pulsar-WD systems lie in the range $1.5-1.7 M_{\odot}$, with a median value of $\sim 1.6 M_{\odot}$ (ignoring PSR J0751+1807 due to the large uncertainties in its mass measurements; Stairs 2004, and references therein). Taking into account the considerable uncertainties, we conservatively choose a range of $1.2 M_{\odot} \leq m_{1} \leq 1.8 M_{\odot}$ for the pulsar mass of PSR J2145-0750. As discussed in Sect. 5.4, this results in allowed companion masses of $0.5 M_{\odot} \leq m_{2} \leq 1.0 M_{\odot}$. We hence conclude that the pulsar companion is a CO-core WD in the intermediate WD mass range. For the median pulsar mass of $\sim 1.6 M_{\odot}$ and the observed median inclination angle of $46^{\circ}$ we obtain a median companion mass of $\sim 0.7 M_{\odot}$.

Using the measured parallax distance and the constraints coming from photometrical observations of the WD companion of PSR J2145-0750, the WD properties can be further constrained. Our analysis reveals that the companion has a mass in the range $0.7 M_{\odot} \leq m_{2} \leq 1.0 M_{\odot}$, a cooling age of $\tau_{\text {cool }}=3.6(2) \mathrm{Gyr}$, and an effective temperature of $T_{\text {eff }}=$ $5750 \pm 600 \mathrm{~K}$, in good agreement with results from previous studies (Lundgren et al. 1996; Hansen \& Phinney 1998; Richer et al. 2000). In particular, the WD model for a companion mass of $0.7 M_{\odot}$, the median value derived from the timing analysis, exactly reproduces the measured WD luminosity for $T_{\text {eff }}=5250 \mathrm{~K}$.

The WD cooling age is roughly a factor of 3 lower than the pulsar's characteristic age of $10.4 \mathrm{Gyr}$, which is generally considered as a rough upper limit of the system age (Camilo et al. 1994b). Under the assumption that the pulsar's magnetic field does not decay, we can use the WD cooling age to estimate the initial spin period $P_{0}$ of PSR J2145-0750, by inverting Eq. (2). The Doppler contributions are subtracted as we apply the intrinsic spin-down rate $\dot{P}^{\text {intr }}$. For the derived system age of $\tau=\tau_{\mathrm{WD}}=3.6(2) \mathrm{Gyr}$ and typical braking indices $n=2 \ldots 3$ (Lyne 1996, and references herein) we hence obtain an initial spin period of $P_{0}=13.0(5) \mathrm{ms}$, which is very close to the current period. Theories regarding the spin-up of recycled pulsars to millisecond periods (Smarr \& Blandford 1976; Ghosh 1995, and references therein) predict that the initial period should be equal to the equilibrium spin period of the neutron star in the X-ray binary phase, accreting at the Eddington rate $\dot{M}_{\text {Edd }}$. For PSR J2145-0750 we find $\dot{M} / \dot{M}_{\text {Edd }}=0.004(1)$. Hence the final accretion rate of the pulsar was highly sub-Eddington, which has also been observed in other IMBP systems (Lundgren et al. 1996). This is not surprising as the unstable CE phase of the binary was too short to spin up the pulsar to the equilibrium spin period. Instead, as proposed by van den Heuvel (1994), the initial period may have resulted from sub-Eddington accretion from the stellar wind of the giant star before reaching the asymptotic giant branch phase.

The prospects for measuring the pulsar mass of the PSR J2145-0750 system are quite low. As the pulsar companion is bright enough in the optical, the system masses could in principle be determined by a spectroscopic study (see van Kerkwijk et al. 1996). However, given the composition of the companion, suitable lines might not be present. Similarly, post-Keplerian effects beyond Shapiro delay will not be detectable in the foreseeable future. As mentioned in Sect. 4.5, relativistic effects such as orbital period decay or periastron advance are fully absorbed by the redefinition of the binary 
period and therefore not observable in this low-eccentricity binary. The precision of the Shapiro delay measurement (or limit) cannot be improved drastically with existing radio telescopes. It will need telescopes like the Square-Kilometre-Array (SKA) to provide a large sample of precision mass measurements.

Acknowledgements. We are very grateful to all staff at the Effelsberg and Jodrell Bank observatories for their help with the observations. We thank Oleg Doroshenko, Christoph Lange, and Norbert Wex for their assistance in the Effelsberg timing project. We would also like to thank the referee for useful suggestions that helped to improve the text. O.L. was partially funded during this work by the European Commission, Marie Curie Training Site programme, under contract No. HPMT-CT-2000-00069.

\section{References}

Alpar, M. A., Cheng, A. F., Ruderman, M. A., \& Shaham, J. 1982, Nature, 300, 728

Arzoumanian, Z., Nice, D. J., Taylor, J. H., \& Thorsett, S. E. 1994, ApJ, 422, 671

Backer, D. C., Dexter, M. R., Zepka, A., et al. 1997, PASP, 109, 61

Backer, D. C., Hama, S., \& Hook, S. V. 1993, ApJ, 404, 636

Bailes, M., Harrison, P. A., Lorimer, D. R., et al. 1994, ApJ, 425, L41

Bailes, M., Ord, S. M., Knight, H. S., \& Hotan, A. W. 2003, ApJ, 595, L49

Bergeron, P., Saumon, D., \& Wesemael, F. 1995, ApJ, 443, 764

Bhattacharya, D., \& van den Heuvel, E. P. J. 1991, Phys. Rep., 203, 1

Blöcker, T., Herwig, F., Driebe, T., Bramkamp, H., \& Schönberner, D. 1997, in White dwarfs, ed. J. Isern, M. Hernanz, \& E. Garcia-Berro, ASSL, 214, 57

Blöcker, T. 1995, A\&A, 299, 755

Camilo, F., Foster, R. S., \& Wolszczan, A. 1994a, ApJ, 437, L39

Camilo, F., Thorsett, S. E., \& Kulkarni, S. R. 1994b, ApJ, 421, L15

Cordes, J. M., \& Lazio, T. J. W. 2002, ApJ, submitted [arXiv: astro-ph/0207156]

Damour, T., \& Taylor, J. H. 1991, ApJ, 366, 501

Damour, T., \& Taylor, J. H. 1992, Phys. Rev. D, 45, 1840

Doroshenko, O., Löhmer, O., Kramer, M., et al. 2001, A\&A, 379, 579

Downs, G. S., \& Reichley, P. E. 1983, ApJS, 53, 169

Ghosh, P. 1995, JA\&A, 16, 289

Gould, D. M., \& Lyne, A. G. 1998, MNRAS, 301, 235

Hankins, T. H., \& Rickett, B. J. 1975, in Methods in Computational Physics, Radio Astronomy (New York: Academic Press), 14, 55

Hansen, B. M. S., \& Phinney, E. S. 1998, MNRAS, 294, 569

Hobbs, G., Faulkner, A., Stairs, I. H., et al. 2004, MNRAS, 352, 1439

Jacoby, B. A., Bailes, M., van Kerkwijk, M. H., et al. 2003, ApJ, 599, L99

Johnston, S., Nicastro, L., \& Koribalski, B. 1998, MNRAS, 297, 108

Kaspi, V. M., Taylor, J. H., \& Ryba, M. 1994, ApJ, 428, 713

Kassim, N. E., \& Weiler, K. W. 1990, Nature, 343, 146

Kopeikin, S. M. 1996, ApJ, 467, L93

Kramer, M., Xilouris, K. M., Jessner, A., et al. 1997, A\&A, 322, 846
Kramer, M., Xilouris, K. M., Lorimer, D. R., et al. 1998, ApJ, 501, 270

Kuijken, K., \& Gilmore, G. 1989, MNRAS, 239, 571

Lange, C., Camilo, F., Wex, N., et al. 2001, MNRAS, 326, 274

Löhmer, O., Lewandowski, W., Wolszczan, A., \& Wielebinski, R. 2004, ApJ, submitted

Lommen, A. N. 2002, in Neutron Stars, Pulsars, and Supernova Remnants, ed. W. Becker, H. Lesch, \& J. Trümper, 114

Lundgren, S. C., Foster, R. S., \& Camilo, F. 1996, in Pulsars: Problems and Progress, ed. S. Johnston, M. A. Walker, \& M. Bailes (San Francisco: ASP), IAU Coll., 160, 497

Lyne, A. G. 1996, in Pulsars: Problems and Progress, ed. S. Johnston, M. A. Walker, \& M. Bailes (San Francisco: ASP), IAU Coll., 160,73

Lyne, A. G., Burgay, M., Kramer, M., et al. 2004, Science, 303, 1153

Monet, D. G., Dahn, C. C., Vrba, F. J., et al. 1992, AJ, 103, 638

Nicastro, L., \& Johnston, S. 1995, MNRAS, 273, 122

Nice, D. J. 2003, in Young Neutron Stars and Their Environments, ed. F. Camilo, \& B. M. Gaensler (San Francisco: ASP), IAU Symp., 218,175

Nice, D. J., Splaver, E. M., \& Stairs, I. H. 2001, ApJ, 549, 516

Nice, D. J., Splaver, E. M., \& Stairs, I. H. 2003, in Radio Pulsars, ed. M. Bailes, D. J. Nice, \& S. E. Thorsett, ASP Conf. Ser., 302, 75

Phinney, E. S., \& Kulkarni, S. R. 1994, ARA\&A, 32, 591

Radhakrishnan, V., \& Cooke, D. J. 1969, Astrophys. Lett., 3, 225

Reid, M. J. 1993, ARA\&A, 31, 345

Richer, H. B., Hansen, B., Limongi, M., et al. 2000, ApJ, 529, 318

Ryba, M. F., \& Taylor, J. H. 1991, ApJ, 371, 739

Sallmen, S. 1998, Ph.D. Thesis, University of California at Berkeley

Sandhu, J. S., Bailes, M., Manchester, R. N., et al. 1997, ApJ, 478, L95

Seiradakis, J. H., Gil, J. A., Graham, D. A., et al. 1995, A\&AS, 111, 205

Shapiro, I. I. 1964, Phys. Rev. Lett., 13, 789

Shklovskii, I. S. 1970, SvA, 13, 562

Smarr, L. L., \& Blandford, R. 1976, ApJ, 207, 574

Splaver, E. M., Nice, D. J., Arzoumanian, Z., et al. 2002, ApJ, 581, 509

Stairs, I. H. 2004, Science, in press

Stairs, I. H., Thorsett, S. E., \& Camilo, F. 1999, ApJS, 123, 627

Standish, E. M. 1990, A\&A, 233, 252

Taam, R. E., King, A. R., \& Ritter, H. 2000, ApJ, 541, 329

Tauris, T. M. 1996, A\&A, 315, 453

Tauris, T. M., van den Heuvel, E. P. J., \& Savonije, G. J. 2000, ApJ, 530, L93

Taylor, J. H., \& Cordes, J. M. 1993, ApJ, 411, 674

Thorsett, S. E., \& Chakrabarty, D. 1999, ApJ, 512, 288

Toscano, M., Sandhu, J. S., Bailes, M., et al. 1999, MNRAS, 307, 925

van den Heuvel, E. P. J. 1994, A\&A, 291, L39

van Kerkwijk, M. H., Bergeron, P., \& Kulkarni, S. R. 1996, ApJ, 467, L89

van Straten, W., Bailes, M., Britton, M., et al. 2001, Nature, 412, 158

Xilouris, K. M., Kramer, M., Jessner, A., et al. 1998, ApJ, 501, 286 